\title{
Suppression of Laser Nonuniformity Imprinting Using a Thin High-Z Coating
}

\author{
Max Karasik, ${ }^{1}$ J. L. Weaver, ${ }^{1}$ Y. Aglitskiy, ${ }^{2}$ J. Oh, ${ }^{3}$ and S. P. Obenschain ${ }^{1}$ \\ ${ }^{1}$ Plasma Physics Division, Naval Research Laboratory, Washington, D.C. 20375, USA \\ ${ }^{2}$ Leidos, Inc., 11951 Freedom Drive Reston, VA 20190, USA \\ ${ }^{3}$ RSI, 4325-B Forbes Boulevard, Lanham, Maryland 20706, USA
}

(Received 30 April 2014; revised manuscript received 20 November 2014; published 26 February 2015)

\begin{abstract}
Imprinting of laser nonuniformity is a limiting factor in direct-drive inertial confinement fusion experiments, particularly when available laser smoothing is limited. A thin ( $400 \AA)$ high- $Z$ metal coating is found to substantially suppress laser imprint for planar targets driven by pulse shapes and intensities relevant to implosions on the National Ignition Facility while retaining low adiabat target acceleration. A hybrid of indirect and direct drive, this configuration results in initial ablation by $\mathrm{x}$ rays from the heated high- $Z$ layer, creating a large standoff for perturbation smoothing.
\end{abstract}

Hydrodynamic instability seeded by laser nonuniformity (laser imprint) is an important factor in the performance of direct-drive inertial confinement fusion (ICF) targets. Though modern beam smoothing techniques [1,2] can reduce time-averaged single beam nonuniformity down to $\sim 1 \%$, residual nonuniformity amplified by Raleigh-Taylor (RT) and Richtmyer-Meshkov instabilities can reduce the fusion yield or spoil the ignition. Furthermore, the current ignition-scale laser, the National Ignition Facility (NIF), is projected to have only limited beam smoothing capability [3]. Various imprint mitigation strategies have been proposed, including the use of foam-buffered targets [4], preformed plasma [5,6], and high- $Z$ doped ablators [7]. A relatively simple yet highly effective method demonstrated in Ref. [8] uses a thin $(<1000 \AA)$ high-Z (Au or Pd) overcoat on the laser side of the target. Most of the laser imprint is expected to occur during the initial lowerintensity part of the laser pulse $[9,10]$, which generates the first shocks necessary to compress the target to achieve high gain [11]. The overcoat initially absorbs the laser and emits soft $\mathrm{x}$ rays that ablate the underlying target. This initially x-ray drive allows a large standoff distance between laser absorption and ablation and gives a higher ablation velocity [12], resulting in smoothing of laser perturbations [13] and higher ablative stabilization [14]. The coating is sufficiently thin so that it becomes transparent to the main part of the pulse, at which point the laser ablates the target directly and $\mathrm{x}$-ray preheat is minimized. The previous experiments utilized a low intensity $\left(1-10 \mathrm{TW} / \mathrm{cm}^{2}\right)$ "foot" to set the target on a low adiabat followed by the main pulse. Current ICF target designs [3] shape the adiabat using higher intensity $\left(\leq 10^{14} \mathrm{~W} / \mathrm{cm}^{2}\right)$, short duration ( $\left.\gtrsim 100 \mathrm{ps}\right)$ spikes followed by the main drive. The effect of the spike without the high- $Z$ coating on the imprint and RT growth mitigation via spatial adiabatic shaping have been studied in numerous works [15-19]. The higher intensities and shorter durations of the spikes could affect the x-ray spectrum generated by the overcoat and the overcoat dynamics. The experiments reported here show for the first time that the high$Z$ coating method of imprint suppression works with such spike pulse shapes and intensities, with a view to utilization for direct drive on ignition-scale facilities, such as the NIF.

Planar $50 \mu \mathrm{m}$ polystyrene $(\mathrm{CH})$ foils uncoated or with $\mathrm{Au}$ or Pd on the laser side were driven by the Nike $\mathrm{KrF}$ $(\lambda=248 \mathrm{~nm})$ laser [20] with a pulse shape shown in Fig. 1. The pulse shape consists of a 400 ps FWHM spike followed by a 3 ns main pulse. Peak intensities of the spike and main pulse $\left(0.5 \times 10^{14}\right.$ and $\left.2 \times 10^{14} \mathrm{~W} / \mathrm{cm}^{2}\right)$ are comparable to those of the spike and the first step of the main pulse of the ignition design of Ref. [3] $\left(1 \times 10^{14}\right.$ and $\left.2 \times 10^{14} \mathrm{~W} / \mathrm{cm}^{2}\right)$. For the $50 \mu \mathrm{m}$ thick $\mathrm{CH}$, the pulse shape results in a near simultaneous breakout of the two shocks launched by the spike and the main drive at the rear surface. Laser spot size zooming [21] was utilized so that the spike diameter was $1060 \mu \mathrm{m}$ and the drive pulse diameter was $440 \mu \mathrm{m}$. Areal

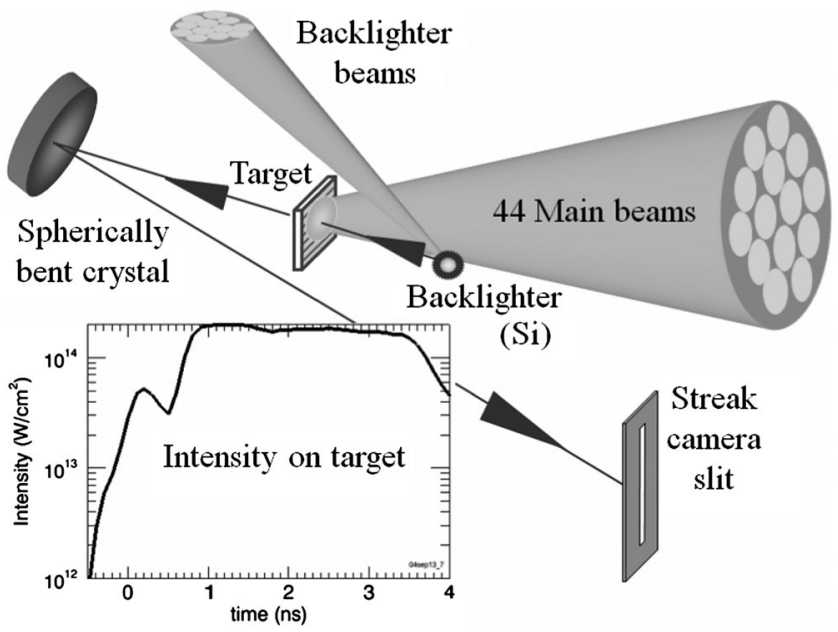

FIG. 1. Laser intensity on target (inset) and the experimental setup showing face-on radiography using monochromatic x-ray imaging. 


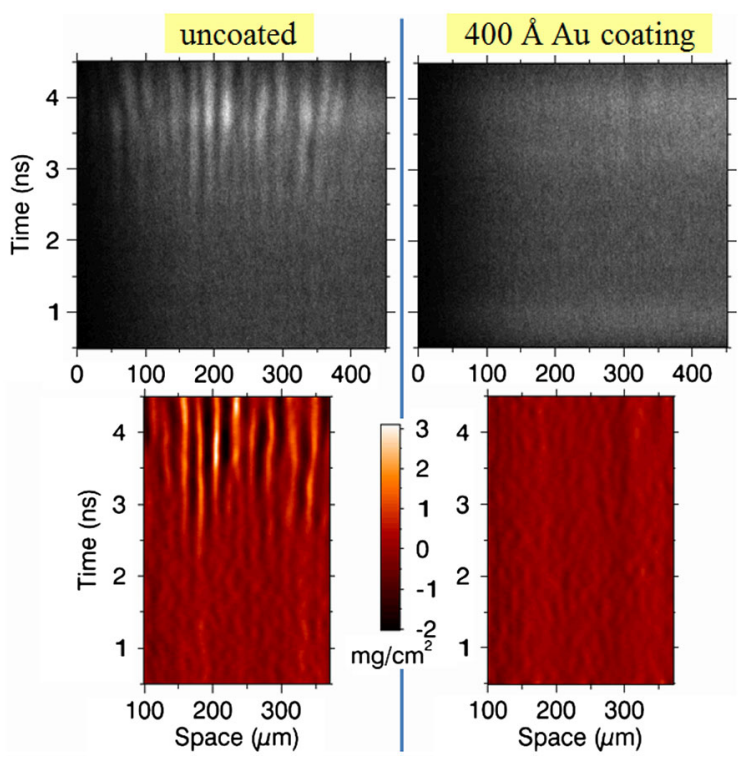

FIG. 2 (color online). Raw streaked face-on radiographs with $1.86 \mathrm{keV} x$ rays (top row) and the resulting areal mass fluctuations in the range of 10-100 $\mu \mathrm{m}$ (bottom row) for uncoated (left) and $400 \AA$ Au coated (right) targets. The coating suppresses the imprint to below the measurement noise level.

mass nonuniformity was imprinted by the residual nonuniformity of the induced spatial incoherence (ISI) smoothed beams [1] during the spike and amplified by the RT instability during the acceleration driven by the main portion of the pulse. It was measured by radiographing the target face on (Fig. 1) using a Si backlighter and monochromatic curved crystal imaging at $1.86 \mathrm{keV}$ coupled to an $\mathrm{x}$-ray streak camera [22]. The angle of the radiography is $25^{\circ}$ with respect to target normal. Magnification is $14.6 \times$ and the streak camera slit is $200 \mu \mathrm{m}$, giving an equivalent slit width of $14 \mu \mathrm{m}$ at the target plane. Streaked radiographs were processed to correct for the spatial resolution of the imaging system using Wiener filtering [23] with the modulation transfer function measured in situ using a knife-edge target. Areal mass fluctuations were obtained from the corrected radiographs using the cold $\mathrm{CH}$ opacity of $346 \mathrm{~cm}^{2} / \mathrm{g}$ at $1.86 \mathrm{keV}$ [24] and bandpass filtered to retain spatial frequencies in the range 10-100 $\mu \mathrm{m}$.

Figure 2 shows examples of raw streaked radiographs and processed areal mass fluctuations for an uncoated and a $400 \AA$ Au coated target. Both raw and processed images show measurable RT-amplified imprint starting at approximately $2.5 \mathrm{~ns}$ for the uncoated case, and only measurement-noise-level fluctuations in the coated case.

Figure 3 shows that $\mathrm{Pd}$ also suppresses the imprint, although about $600 \AA \mathrm{Pd}$ is required to achieve the effect seen for $400 \AA \mathrm{Au}$. The intermediate case of $400 \AA \mathrm{Pd}$ shows some imprint reduction.

Figure 4 shows the time evolution of the rms of areal mass perturbations for uncoated and coated targets obtained from the shots shown in Figs. 2 and 3 as well as a case with $200 \AA \mathrm{Au}$. The two uncoated examples show significant variation in the later, nonlinear evolution. The

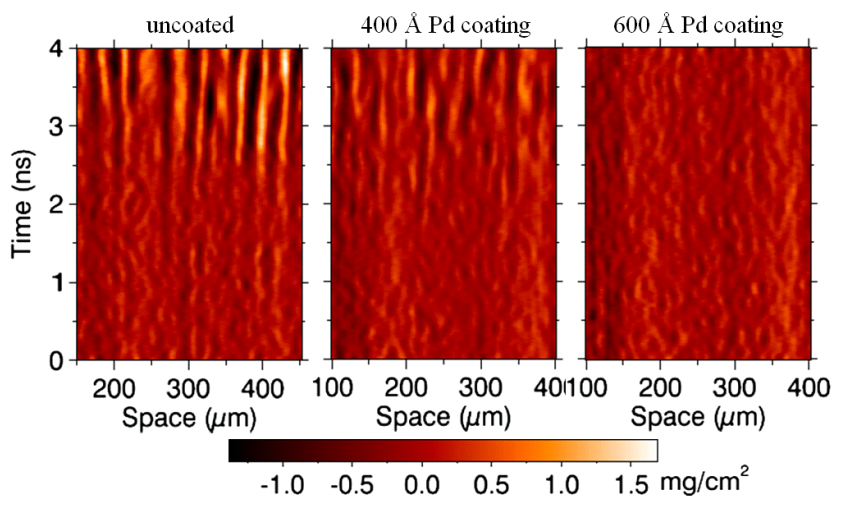

FIG. 3 (color online). Areal mass fluctuations for uncoated, $400 \AA \mathrm{Pd}$, and $600 \AA \mathrm{Pd}$ coated targets. For the $600 \AA$ coating, the imprint is reduced to below measurement noise.

initial growth of the fluctuations is reproducible, however. For coating thicknesses of $400 \AA \mathrm{Au}$ and $600 \AA \mathrm{Pd}$, the imprint never rises above the measurement noise level. The thinner $\mathrm{Au}$ and $\mathrm{Pd}$ coatings show some imprint reduction.

Exposing a higher- $Z$ material to the laser can result in an $\mathrm{X}$-ray preheat of the rest of the ablator or of the fuel in an ICF pellet. Preheat of the outer layers of the ablator can be beneficial in that it can reduce the RT growth rate at the ablation front $[11,25]$. Excessive preheat, however, can cause instability growth at the ablator-fuel interface or can preheat the fuel itself, leading to a spoiled implosion. To test whether the lack of a RT-amplified imprint in the coated case is due to a reduced RT growth rate, coated and uncoated targets with a preimposed ripple on the laser side were shot, as shown in Fig. 5. Both cases show strong growth of the preimposed ripple, indicating that the coating, in fact, suppresses the imprint and does not have a significant effect on the RT growth.

A more detailed comparison of the ripple growth for the two shots is shown in Fig. 6, which gives a lineout of the

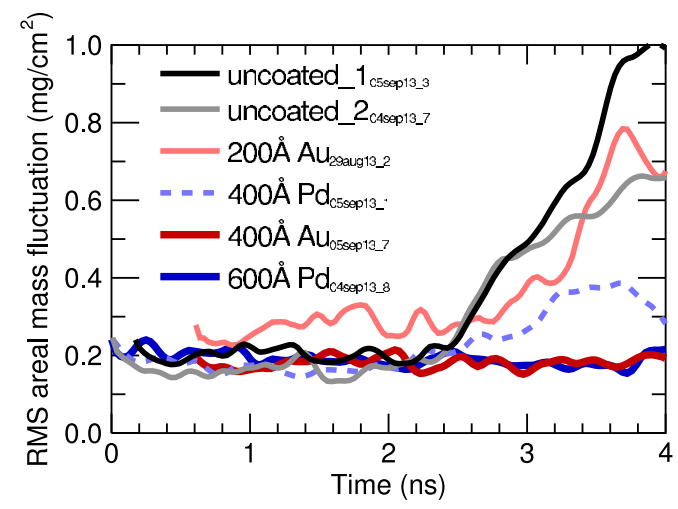

FIG. 4 (color online). rms of areal mass perturbations vs time for uncoated, $200 \AA \mathrm{Au}, 400 \AA \mathrm{Pd}, 400 \AA \mathrm{Au}$, and $600 \AA \mathrm{Pd}$ overcoat targets. Two uncoated shots are shown with larger and smaller rms values in the nonlinear stage of growth, representative of extremes of shot-to-shot variation at that stage. Baseline noise level for the measurement is generally $0.2 \mathrm{mg} / \mathrm{cm}^{2}$, except for the $200 \AA \mathrm{Au}$ case due to weaker backlighting on that shot. 


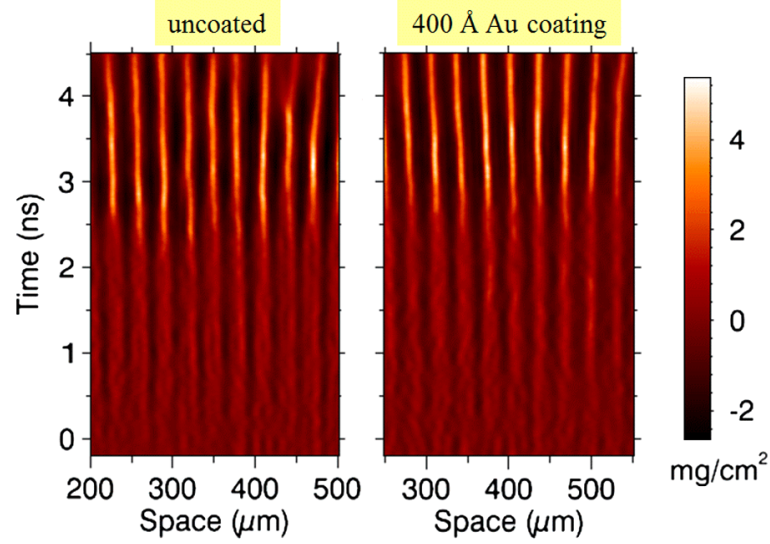

FIG. 5 (color online). Evolution of areal mass perturbations started by a front surface ripple $(0.5 \mu \mathrm{m}$ amplitude, $30 \mu \mathrm{m}$ wavelength) for uncoated and $400 \AA \mathrm{Au}$ overcoat targets. The ripple development is very similar in both cases.

rms areal mass perturbation. It can be seen that in the coated case, the RT growth appears delayed by approximately $0.3 \mathrm{~ns}$, but the growth rate is not significantly affected.

Another indication of whether the target is being excessively preheated is the motion of the rear surface prior to the shock breakout. For this observation, an additional backlighter-crystal pair radiographed the target side on, an example of which is shown for a $600 \AA \mathrm{Pd}$ coated target in Fig. 7. As can be seen in the figure, no motion of the rear surface is evident prior to the shock breakout. However, because of the large lateral extent of the target $(\approx 2 \mathrm{~mm})$ and a slight angle of the diagnostic axis to the plane of the target, the target shadow is $60 \mu \mathrm{m}$, rather than the $50 \mu \mathrm{m}$ target thickness. Therefore some shadowing of the rear surface is possible, and it can only be concluded that prior to the shock breakout the center portion of the rear surface did not move more than $5 \mu \mathrm{m}$. Based on this, a crude estimate of an upper bound on preheat can be made as follows. Expansion of no more than $5 \mu \mathrm{m}$ over $1.5 \mathrm{~ns}$

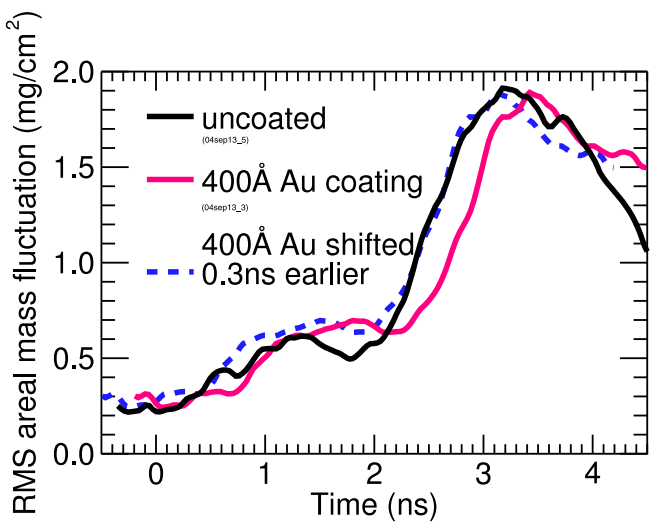

FIG. 6 (color online). rms of areal mass perturbations for the shots shown in Fig. 5. The RT growth rate is unchanged by the coating, except for an approximately $0.3 \mathrm{~ns}$ time delay (for comparison, the dashed line shows the coated case shifted 0.3 ns earlier). gives an upper limit of expansion velocity of $3.3 \mathrm{~km} / \mathrm{s}$. Assuming expansion at sonic speed, we can estimate the preheat as the energy density needed for this sound speed in polystyrene. Shock compression data [26] show that $0.218 \mathrm{~kJ} / \mathrm{g}$ is needed to achieve a $3.38 \mathrm{~km} / \mathrm{s}$ shock speed in polystyrene (corresponding to a $23 \mathrm{kbar}$ shock). Noting that shock is subsonic in the shocked material, this would be an upper limit on the energy density, giving an upper limit on the preheat per unit area for the $50 \mu \mathrm{m}$ thick polystyrene target of $(218 \mathrm{~J} / \mathrm{g})\left(1.07 \mathrm{~g} / \mathrm{cm}^{3}\right)\left(50 \times 10^{-4} \mathrm{~cm}\right)=1.2 \mathrm{~J} / \mathrm{cm}^{2}$. We plan to implement more sensitive and direct diagnostics of a preheat in future experiments. Detailed simulations and experiments are needed to assess the effect of such a preheat on an ignition pellet, as plastic and the cryogenic fuel have such different X-ray opacities.

Further insight into the mechanism of imprint suppression can be gained by examining $\mathrm{x}$-ray emission spectra. Three cases of time-resolved x-ray spectra in the 5-40 $\AA$ range are shown in Fig. 8. For both $\mathrm{Au}$ and $\mathrm{Pd}$ coated targets, soft $\mathrm{x}$ rays peak soon after the start of the main pulse, then decay to the level of the uncoated plastic target emission during the pulse, consistent with initially $\mathrm{x}$-raydriven ablation transitioning to direct drive.

The large separation allowed by the initially $\mathrm{x}$-ray drive that would give a smoothing of laser perturbations is evident in side-on shadowgraphy of the high- $Z$ overcoat [28]. As can be seen in Fig. 9, the high- $Z$ layer is expanded and is pushed off the original target surface by the ablating $\mathrm{CH}$. Prior to the start of the laser pulse, the high- $Z$ coating, due to its low thermal mass, is already preexpanded by a low intensity $\left(\sim 10^{7} \mathrm{~W} / \mathrm{cm}^{2}\right), \sim 100 \mathrm{~ns}$ duration prepulse [29] present on target on Nike. In the case of the Au overcoat, the coating is pushed off the original target surface, while Pd hugs the surface until the start of the laser pulse. Imprint suppression is observed in both cases, however, indicating that it may not be very sensitive to the initial condition.

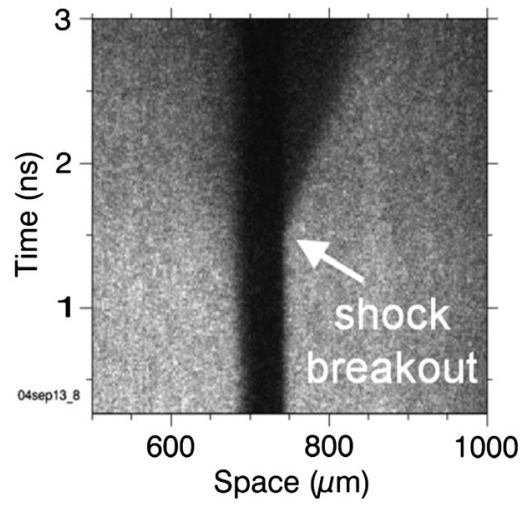

FIG. 7. Streaked side-on backlighting at $1.86 \mathrm{keV}$ of a $600 \AA \mathrm{Pd}$ coated target. The laser is coming from the left. Though the target is $50 \mu \mathrm{m}$ thick, the shadow prior to shock breakout is $60 \mu \mathrm{m}$ wide due to a slight rotation with respect to the diagnostic plane and the $\approx 2 \mathrm{~mm}$ width of the target. No motion of the rear of the target is evident prior to the shock breakout. 

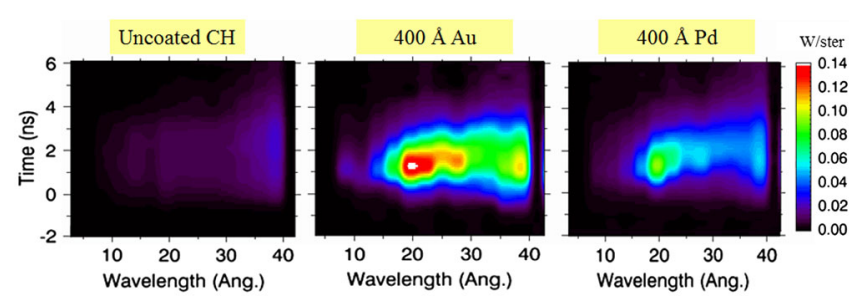

FIG. 8 (color online). Absolutely calibrated x-ray spectra show a marked increase in soft x-ray emission for $\mathrm{Au}$ and $\mathrm{Pd}$ coated targets as compared to the uncoated case. The emission peaks soon after the start of the main pulse (main pulse half rise is at $t=0.7 \mathrm{~ns}$ ) for the coated targets, decaying to the level of the uncoated ones during the pulse. The spectra are measured by the individual photodiode channels of a transmission grating spectrometer [27], with an approximately $0.4 \mathrm{~ns}$ time resolution.

The effect of preexpansion on imprint suppression will be investigated in more detail in future work.

Observation of an effective imprint suppression with $400 \AA$ of $\mathrm{Au}$ and $600 \AA$ of $\mathrm{Pd}$ raises the question of what determines the thickness of the coating required to see the effect. Our understanding is that it must be sufficiently thick so that it is initially opaque to the laser and also
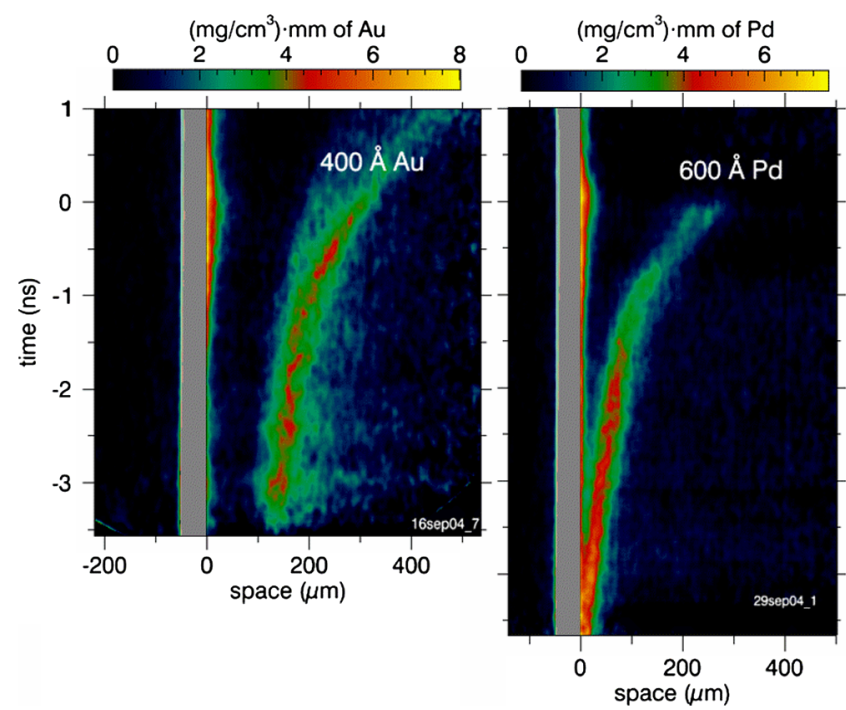

FIG. 9 (color online). Large separation between the high- $Z$ layer and the underlying $\mathrm{CH}$ as evidenced from side-on radiography at $1.86 \mathrm{keV}$. Shown is the line-integrated density of Au (left panel) and Pd (right panel) prior to and in the early stages of the laser pulse. The line density is obtained from absorption of the $1.86 \mathrm{keV} x$ rays in the high- $Z$ layer (the ablated $\mathrm{CH}$ opacity is too low to be seen; Au and Pd lose opacity once the main drive comes on). These shots had pulse shape with a $4 \mathrm{TW} / \mathrm{cm}^{2}$ foot starting at $t=-2 \mathrm{~ns}$ and a $1 \times 10^{14} \mathrm{~W} / \mathrm{cm}^{2}$ main drive at $t=0$. During the pulse, the ablation of the underlying $\mathrm{CH}$ moves the high- $Z$ layer toward the laser (coming in from the right). Both $\mathrm{Au}$ and $\mathrm{Pd}$ are preexpanded by the low-level prepulse prior to the start of the laser pulse; while Pd hugs the original target surface (the shadow of which is shown as the gray vertical strip) prior to the laser pulse, $\mathrm{Au}$ is pushed off by the underlying $\mathrm{CH}$ vaporization. Imprint suppression is effective in both cases, however. generates sufficient soft $\mathrm{x}$-ray flux so that ablation proceeds by $\mathrm{x}$ rays rather than by thermal conduction, allowing the large separation between the laser absorption and the ablation for smoothing to take place. Au is expected to be a more efficient radiator because of its higher $Z$, and so the fact that a thinner coating of $\mathrm{Au}$ than $\mathrm{Pd}$ is required is not surprising. The scaling for different elements would depend on the details of atomic physics of the laser absorption as the initially metallic coating is heated, and the non local-thermodynamic-equilibrium radiation physics once it is hot. We are in the process of improving our simulation codes to help understand these physics further.

In summary, the thin overcoat of a high- $Z$ material, Au or $\mathrm{Pd}$, has been shown to substantially suppress laser imprint without significant preheat. A laser with a high degree of smoothing like the ISI-smoothed $\mathrm{KrF}$ may or may not require additional imprint mitigation for direct drive. For the NIF, however, where laser beam smoothing is insufficient for most direct-drive experiments, the high- $Z$ coating approach may enable a range of new experiments using direct drive. If successfully ported to the laser conditions there, it would not only improve planar experiments but may also enable higher performance direct-drive fusion capsule implosions there. High- $Z$ overcoat was already successfully used to increase the yield of implosions on the OMEGA tripled Nd:glass laser [30]. In order to use the high- $Z$ coating on the NIF ignition pellets, the details of the triple-picket pulse shape design of Ref. [3] would need to be modified using guidance from simulations to maintain proper adiabatic shaping and shock timing. Our group performed initial simulations of a shock ignition design for a NIF class ( $850 \mathrm{~kJ}, 0.351 \mu \mathrm{m}$ laser wavelength) target with a $400 \AA$ Au coating that gives a 1D gain of 80 [31]. Detailed experiments are planned on the OMEGA EP and NIF lasers to adapt the technique to implosions on the NIF. Looking toward the inertial fusion energy application, the coating would not only improve implosion performance but may also improve the cryogenic fuel capsule's ability to survive injection into the hot reactor chamber due to the high reflectivity of the metal coating for infrared wavelengths [32].

The authors are grateful for the work of the Nike laser and target crews-D. P. Brown, B. Jenkins, T. J. Kessler, S. Krafsig, and S. Terrell — the assistance of L. Y. Chan, and the helpful discussions with A. L. Velikovich. This work is supported by the U.S. DOE NNSA.

[1] R. H. Lehmberg and S. P. Obenschain, Use of induced spatial incoherence for uniform illumination of laser fusion targets, Opt. Commun. 46, 27 (1983).

[2] S. Skupsky, R. W. Short, T. Kessler, R. S. Craxton, S. Letzring, and J. M. Soures, Improved laser-beam uniformity using the angular dispersion of frequency modulated light, J. Appl. Phys. 66, 3456 (1989).

[3] T. J. B. Collins, J. A. Marozas, K. S. Anderson, R. Betti, R. S. Craxton, J. A. Delettrez, V. N. Goncharov, D. R. Harding, F. J. Marshall, R. L. McCrory et al., A polar-drive-ignition 
design for the National Ignition Facility, Phys. Plasmas 19, 056308 (2012).

[4] M. Desselberger, M. W. Jones, J. Edwards, M. Dunne, and O. Willi, Use of X-ray Preheated Foam Layers to Reduce Beam Structure Imprint in Laser-Driven Targets, Phys. Rev. Lett. 74, 2961 (1995).

[5] M. Desselberger, T. Afshar-rad, F. Khattak, S. Viana, and O. Willi, Nonuniformity Imprint on the Ablation Surface of Laser-Irradiated Targets, Phys. Rev. Lett. 68, 1539 (1992).

[6] M. Nishikino, H. Shiraga, N. Miyanaga, N. Ohnishi, K. Shigemori, S. Fujioka, M. Nakai, H. Nishimura, H. Azechi, $\mathrm{K}$. Mima et al., Imprint reduction in a plasma layer preformed with X-ray irradiation, Phys. Plasmas 9, 1381 (2002).

[7] S. X. Hu, G. Fiksel, V. N. Goncharov, S. Skupsky, D. D. Meyerhofer, and V. A. Smalyuk, Mitigating Laser Imprint in Direct-Drive Inertial Confinement Fusion Implosions with High-Z Dopants, Phys. Rev. Lett. 108, 195003 (2012).

[8] S. P. Obenschain, D. G. Colombant, M. Karasik, C. J. Pawley, V. Serlin, A. J. Schmitt, J. L. Weaver, J. H. Gardner, L. Phillips, Y. Aglitskiy et al., Effects of thin high-Z layers on the hydrodynamics of laser-accelerated plastic targets, Phys. Plasmas 9, 2234 (2002).

[9] M. H. Emery, J. H. Gardner, R. H. Lehmberg, and S. P. Obenschain, Hydrodynamic target response to an induced spatial incoherence-smoothed laser beam, Phys. Fluids B 3, 2640 (1991).

[10] V. A. Smalyuk, V. N. Goncharov, T. R. Boehly, J. A. Delettrez, D. Y. Li, J. A. Marozas, A. V. Maximov, D. D. Meyerhofer, S. P. Regan, and T. C. Sangster, Measurements of laser-imprinting sensitivity to relative beam mistiming in planar plastic foils driven by multiple overlapping laser beams, Phys. Plasmas 12, 072703 (2005).

[11] S. E. Bodner, D. G. Colombant, J.H. Gardner, R. H. Lehmberg, S. P. Obenschain, L. Phillips, A. J. Schmitt, J. D. Sethian, R. L. McCrory, Q. Seka et al., Direct-drive laser fusion: Status and prospects, Phys. Plasmas 5, 1901 (1998).

[12] S. Atzeni and J. Meyer-ter-Vehn, The Physics of Inertial Fusion (Clarendon Press, Oxford, 2004).

[13] V. N. Goncharov, S. Skupsky, T. R. Boehly, J. P. Knauer, P. McKenty, V. A. Smalyuk, R. P. J. Town, O. V. Gotchev, R. Betti, and D. D. Meyerhofer, A model of laser imprinting, Phys. Plasmas 7, 2062 (2000).

[14] S. E. Bodner, Rayleigh-Taylor Instability and Laser-Pellet Fusion, Phys. Rev. Lett. 33, 761 (1974).

[15] N. Metzler, A. L. Velikovich, A. J. Schmitt, and J. H. Gardner, Laser imprint reduction with a short shaping laser pulse incident upon a foam-plastic target, Phys. Plasmas 9, 5050 (2002).

[16] V. N. Goncharov, J. P. Knauer, P. W. McKenty, P. B. Radha, T. C. Sangster, S. Skupsky, R. Betti, R. L. McCrory, and D. D. Meyerhofer, Improved performance of direct-drive inertial confinement fusion target designs with adiabat shaping using an intensity picket, Phys. Plasmas 10, 1906 (2003).

[17] A. L. Velikovich, A. J. Schmitt, N. Metzler, and J.H. Gardner, Strong shock wave and areal mass oscillations associated with impulsive loading of planar laser targets, Phys. Plasmas 10, 3270 (2003).

[18] J. P. Knauer, K. Anderson, R. Betti, T. J. B. Collins, V. N. Goncharov, P. W. McKenty, D. D. Meyerhofer, P. B. Radha, S. P. Regan, T. C. Sangster et al., Improved target stability using picket pulses to increase and shape the ablator adiabata, Phys. Plasmas 12, 056306 (2005).
[19] Y. Aglitskiy, M. Karasik, A. L. Velikovich, V. Serlin, J. Weaver, T. J. Kessler, A. J. Schmitt, S. P. Obenschain, N. Metzler, and J. Oh, Observation of Strong Oscillations of Areal Mass in an Unsupported Shock Wave, Phys. Rev. Lett. 109, 085001 (2012).

[20] S. P. Obenschain, S. E. Bodner, D. Colombant, K. Gerber, R. H. Lehmberg, E. A. McLean, A. N. Mostovych, M. S. Pronko, C. J. Pawley, A. J. Schmitt et al., The Nike KrF laser facility: Performance and initial target experiments, Phys. Plasmas 3, 2098 (1996).

[21] D. M. Kehne, M. Karasik, Y. Aglitsky, Z. Smyth, S. Terrell, J. L. Weaver, Y. Chan, R. H. Lehmberg, and S.P. Obenschain, Implementation of focal zooming on the Nike KrF laser, Rev. Sci. Instrum. 84, 013509 (2013).

[22] Y. Aglitskiy, A. L. Velikovich, M. Karasik, V. Serlin, C. J. Pawley, A. J. Schmitt, S. P. Obenschain, A. N. Mostovych, J. H. Gardner, and N. Metzler, Direct Observation of Mass Oscillations Due to Ablative Richtmyer-Meshkov Instability in Plastic Targets, Phys. Rev. Lett. 87, 265001 (2001).

[23] W. H. Press, S. A. Teukolsky, W. T. Vetterling, and B. P. Flannery, Numerical Recipes in FORTRAN: The Art of Scientific Computing, 2nd ed. (Cambridge University Press, New York, 1992).

[24] B. L. Henke, E. M. Gullikson, and J. C. Davis, X-ray interactions: Photoabsorption, scattering, transmission, and reflection at $\mathrm{E}=50-30,000 \mathrm{eV}, Z=1-92$, At. Data Nucl. Data Tables 54, 181 (1993).

[25] D. G. Colombant, S. E. Bodner, A. J. Schmitt, M. Klapisch, J. H. Gardner, Y. Aglitskiy, A. V. Deniz, S. P. Obenschain, C. J. Pawley, V. Serlin et al., Effects of radiation on directdrive laser fusion targets, Phys. Plasmas 7, 2046 (2000).

[26] P. R. Levashov, V. E. Fortov, K. V. Khishchenko, I. N. Lomov, and I. V. Lomonosov, in Shock Compression of Condensed Matter-1997, edited by S. C. Schmidt, D. P. Dandekar, and J. W. Forbes (American Institute of Physics, Melville, NY, 1998), p. 47; shock wave database available at http://www.ihed.ras.ru/rusbank/.

[27] J. Weaver, U. Feldman, J. Seely, G. Holland, V. Serlin, M. Klapisch, D. Columbant, and A. Mostovych, Absolutely calibrated, time-resolved measurements of soft $\mathrm{x}$ rays using transmission grating spectrometers at the Nike laser facility, Phys. Plasmas 8, 5230 (2001).

[28] M. Karasik, Y. Aglitskiy, T. J. Kessler, V. Serlin, M. Klapish, and S. P. Obenschain, "Dynamics of high- $Z$ layers on plastic targets irradiated by Nike KrF laser" (to be published).

[29] M. Karasik, A. N. Mostovych, R. H. Lehmberg, Y. Chan, J. L. Weaver, and S. P. Obenschain, Measurements of lowlevel prepulse on Nike KrF laser, J. Appl. Phys. 98, 053101 (2005).

[30] A. N. Mostovych, D. G. Colombant, M. Karasik, J. P. Knauer, A. J. Schmitt, and J. L. Weaver, Enhanced Direct-Drive Implosions with Thin High-Z Ablation Layers, Phys. Rev. Lett. 100, 075002 (2008).

[31] A. J. Schmitt (private communication).

[32] J. D. Sethian, D. G. Colombant, J. Giuliani, R. H. Lehmberg, M. C. Myers, S. P. Obenschain, A. J. Schmitt, J. Weaver, M. F. Wolford, F. Hegeler et al., The science and technologies for fusion energy with lasers and direct-drive targets, IEEE Trans. Plasma Sci. 38, 690 (2010). 